\title{
Batch Transfer of Zebrafish Embryos Into Multiwell Plates
}

\author{
Xuping Zhang, Member, IEEE, Zhe Lu, Member, IEEE, Danielle Gelinas, Brian Ciruna, and \\ Yu Sun, Senior Member, IEEE
}

\begin{abstract}
This paper reports a prototype cooperative robotic system capable of transferring zebrafish embryos in parallel and depositing a single embryo per well in a 96-well microplate. A cell holding device was developed to trap multiple embryos in a regular pattern. The cell holding device and a microplate were positioned and aligned along multiple axes by the system. Embryo release strategies were systematically studied and compared. Experiments demonstrated that out of the 1056 zebrafish embryos used in experiments (i.e., 44 times parallel transfer into 11 96-well plates), 996 wells were successfully filled with one and only one zebrafish embryo, representing a success rate of $94.3 \%$. Further experiments confirmed that the transferred embryos were able to develop into zebrafish with $100 \%$ survival rate.
\end{abstract}

Note to Practitioners-Biological experiments and drug screen require the transfer of individual zebrafish embryos into standard multiwell microplates. Manually pipetting one and only one embryo into each well is tedious and time consuming. Different from the only commercial system for automated zebrafish embryo transfer, which is based on conventional, expensive flow cytometry, the proof-of-principle system reported in this paper utilizes a cell immobilization device and a cooperative robotic mechanism for parallel transfer of zebrafish embryos. The system architecture and transfer techniques promise efficient, cost-effective zebrafish embryo transfer for applications requiring molecule tests on a high number of cells.

Index Terms-Batch transfer, control and alignment, cooperative robotic mechanism, drug screen, high-throughput screen (HTS), multiwell microplate, zebrafish embryo.

\section{INTRODUCTION}

$\mathbf{Z}$ EBRAFISH has become a widely used model in developmental biology as well as in drug discovery [1]-[3]. Zebrafish embryos are optically transparent, allowing image-based screening and assessment of drug effects on internal organs in vivo. Zebrafish embryos are easy to breed, and can be kept alive in standard multiwell plates for a couple of

Manuscript received September 27, 2010; revised December 22, 2010; accepted February 12, 2011. Date of publication March 14, 2011; date of current version July 07,2011 . This work was supported in part by the Natural Sciences and Engineering Research Council of Canada and in part by the Canada Research Chairs Program. This paper was recommended for publication by Associate Editor F. Arai and Editor K. Bohringer upon evaluation of the reviewers' comments.

X. Zhang, Z. Lu, and Y. Sun are with the Department of Mechanical and Industrial Engineering, University of Toronto, Toronto, ON M5S 3G8, Canada (e-mail: sun@mie.utoronto.ca).

D. Gelinas and B. Ciruna are with the Program in Developmental and Stem Cell Biology, Hospital for Sick Children, Toronto, ON M5G 1X8, Canada.

Color versions of one or more of the figures in this paper are available online at http://ieeexplore.ieee.org.

Digital Object Identifier 10.1109/TASE.2011.2121903 days without the need to add nutrients. Furthermore, zebrafish as an animal is also small in size and requires a low amount of drug compound per assay, making the animals also fit in standard multiwell plates for assaying.

Drug discovery demands screening large libraries of compounds (drug candidates). To meet the need to conduct thousands of assays per day, high-throughput screen (HST) has greatly benefited from the use of robotics and automation technologies [4]-[7]. For HTS with zebrafish, one and only one zebrafish embryo needs to be transferred into each well of multiwell microplates, and reagents and media must also be dispensed into wells of assay microplates with automated systems.

The automation of imaging and analysis of zebrafish embryos in 96-well plates for drug screening has been reported [8]. Significant progress has also been made in the development of robotic systems for dispensing reagents and media into wells of microplates at a high speed with high accuracy [9]. Liquid handling robots are now available from several companies, for example, Tecan and Beckman Coulter [10]. However, existing liquid handling robots are not capable of transferring zebrafish embryos into microplates. The transfer of zebrafish embryos ( $\sim 1.2 \mathrm{~mm}$ in diameter) is still performed manually in most labs [11], which is a tedious and laborious task.

No attempt to automate the transfer of zebrafish embryos was made until recently. A cell-sorting instrument (COPAS $\mathrm{XL}$, Union Biometica) became available on the market for dispensing zebrafish embryos into 96-well microplates. This instrument was developed on the basis of conventional flow cytometer that produces sheath flow to line up zebrafish embryos. Precisely controlled pressure dispenses a single zebrafish embryo into a well of a microplate one at a time. Like flow cytometers, this zebrafish embryo transfer instrument is expensive. Additionally, the instrument transfers embryos serially (one at a time), also motivating us to investigate approaches for parallel embryo transfer.

This paper reports an automated system capable of batch transfer of zebrafish embryos into 96-well microplates, to fill each well with one and only one zebrafish embryo in a parallel manner at a high speed and with a high success rate. A vacuum-based cell holding device and a robotic mechanism were developed for batch transfer of zebrafish embryos. Transfer strategies were described to overcome adhesion forces between embryos and the substrate. A vision-guided alignment approach was developed for embryo transfer with high success rates. Experiments were performed with 1056 zebrafish embryos to quantify the performance of the system and operation strategies. The transferred embryos were cultured inside 


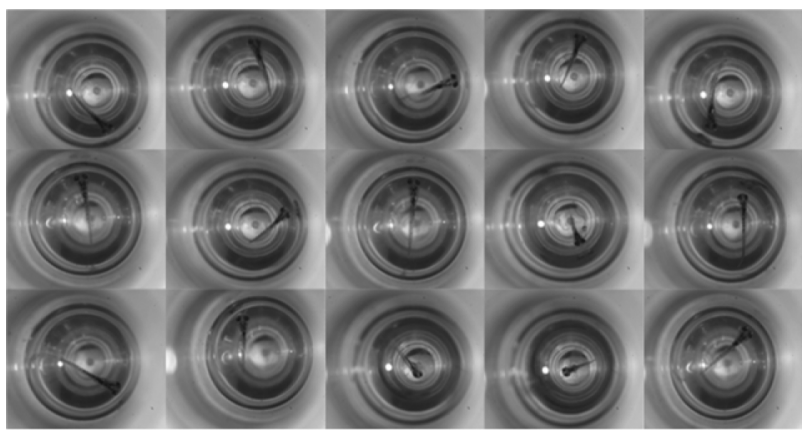

Fig. 1. The system transferred zebrafish embryos into 96-well microplates. Embryos were allowed to develop into zebrafish. Microscopic pictures were stitched together to form this figure, showing a portion of a 96-well microplate. Each well is $5.5 \mathrm{~mm}$ in diameter.

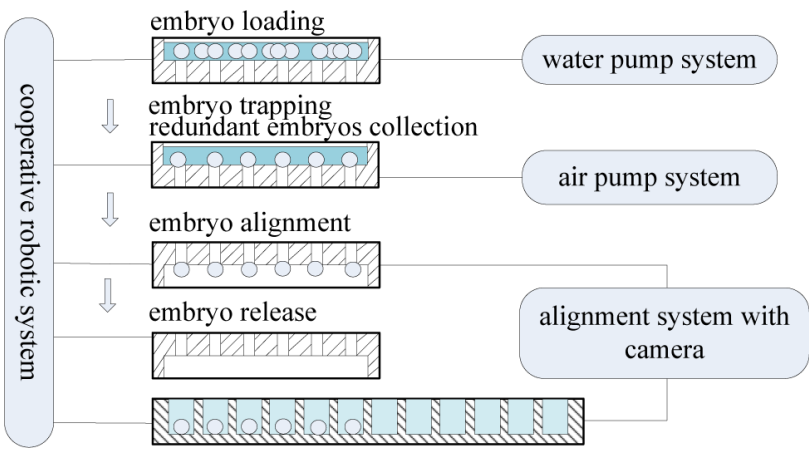

Fig. 2. Schematic showing the operation of the automated zebrafish embryo transfer system at one of four regions. Filling a 96-well plate requires the system to conduct batch transfer of zebrafish embryos four times using the cell holding device with 24 through-holes.

one 96-well microplate and allowed to develop into zebrafish (Fig. 1), demonstrating a development rate of $100 \%$.

\section{TASK ANALYSIS AND System DESIGN}

\section{A. Overall Design}

The overall system and operation procedure are schematically illustrated in Fig. 2. The task is to deposit zebrafish embryos into standard 96-well microplates with each well filled with one and only one embryo. To accomplish the parallel transfer task, our first step is to position and trap many zebrafish embryos into an array on a device. The device is designed such that the trapped cell pattern matches the well array pattern of standard 96-well microplates.

Several technologies of cell positioning/trapping [12] have been reported in recent years, for example, devices based on optical force [13], magnetic force [14], and electrical force [15]. Vacuum-based cell trapping is appealing since low vacuum levels prove effective and do not produce undesired biological complications for further cellular development, as reported in our previous work [16], [17]. Hence, a vacuum-based cell holding device was developed in this work for transferring zebrafish embryos into 96-well microplates. As shown in Fig. 2, a water pump is used to load water to the cell holding device. An air pump system is used to apply negative pressure to immobilize zebrafish embryos and collect redundant embryos and water/culture medium.
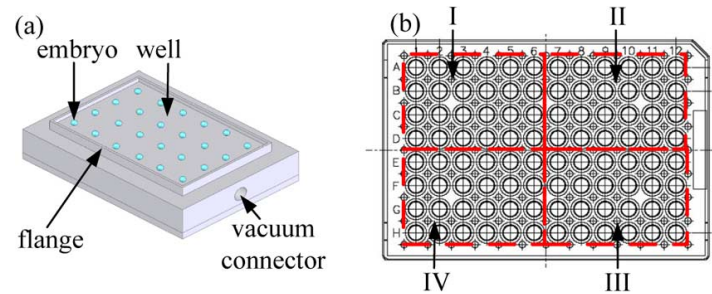

Fig. 3. (a) Cell holding device designed to match the configuration of standard 96-well microplates. The device is capable of immobilizing 24 zebrafish embryos via low vacuum. (b) A standard 96-well plate virtually divided into four subregions.

When embryos are immobilized on the cell holding device, the system "flips" the device upside down and aligns the device with a 96-well microplate (Step 3 in Fig. 2). A robotic mechanism is developed to cooperatively position the cell holding device and the 96-well plate for zebrafish embryo transfer. The number of through-holes on the holding device is important to transfer speed and success rate. A high number of through-holes could shorten the total transfer time since the required number of batch transfer is deceased. However, a larger device size could increases alignment errors of cell holding device and microplate and lead to a low success rate. On the other hand, a low number of through-holes would require more transfers; however, the success rate could be enhanced. Therefore, a device design with 24 through-holes was experimentally chosen in this study to achieve a tradeoff between the operation speed and success rate of embryo transfer. Thus, a 96-well plate is divided into four regions. Within each region, 24 embryos are transferred in parallel. Dividing a plate into four regions reduced the accuracy requirement of the system and increased system reliability. Alignment using visual feedback is integrated into the system to align the cell holding device and the 96-well plate. Finally, embryo release strategies are required to tackle adhesion forces between embryos and the cell holding device substrate in order to successful deposit embryos into the wells.

\section{B. Cell Holding Device}

Microplates such as those with 96 wells are standard tools used in zebrafish screening. Microplates of higher density and lower volume (e.g., 384- or 1536-well plates) can increase the throughput of HTS; however, the small well sizes of the highdensity microplates can limit the development of zebrafish embryos. In this study, 96-well plates are used to prove the concept of parallel embryo transfer. The approach, however, will also be applicable to other standard microplates.

The cell holding device used in this study, as shown in Fig. 3, is designed to match the configuration of standard 96-well microplates (e.g., pitch). The cell device has only 24 through-holes for embryo immobilization in this work. Filling a 96-well plate requires the system to conduct batch transfer of zebrafish embryos four times with the cell holding device. Each subregion on a 96-well plate contains 6 by 4 wells with a well center to center pitch of $9 \mathrm{~mm}$ [Fig. 3(b)].

Fig. 3(a) shows a schematic of the cell holding device. The 6 by 4 through-holes are formed on the top surface and connected to the chamber inside the device. The outlet of the device 


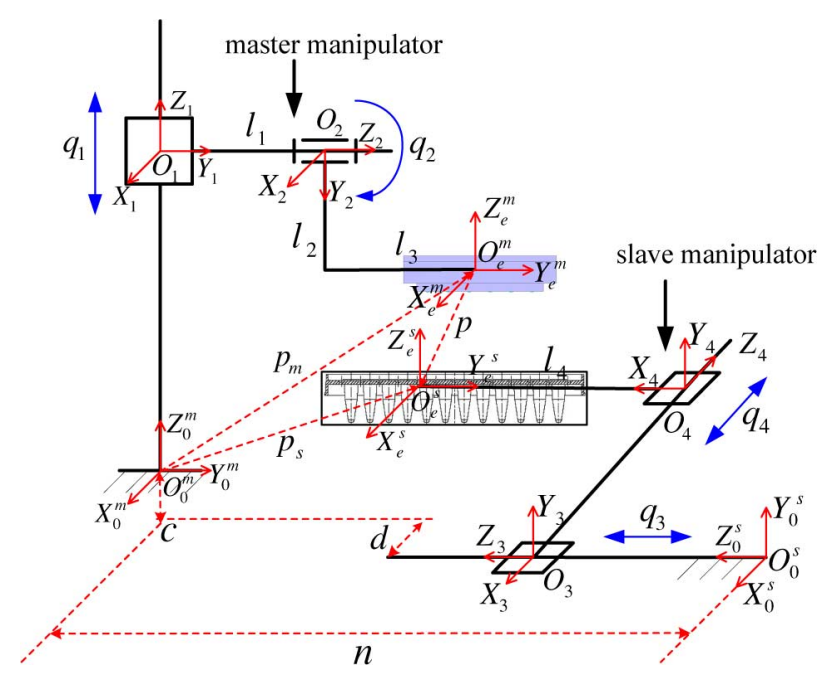

Fig. 4. Coordinates of the multiple-degrees-of-freedom system.

is connected to a precision vacuum pump. The through-holes are designed to be evenly spaced with hole pitches being equal to the well pitch of standard 96-well plates. Considering that average diameter of zebrafish embryos is around $1 \mathrm{~mm}$, the diameter of though-holes is set to be $0.5 \mathrm{~mm}$ so that embryos can be efficiently immobilized on top of the through-holes. A flange with a height of $1.5 \mathrm{~mm}$ is constructed on the device. The dam from the flange accommodates water/culture medium to make all embryos submerged in water and prevent water from spilling out of the device during operation.

\section{Robotic Mechanism Design}

A robotic mechanism is designed to accomplish several functions: 1) to rotate the cell holding device; 2) to move the cell holding device up and down; and 3) to position the 96-well plate. Fig. 4 shows the cooperative robot mechanism with four degrees of freedom, consisting of one master robot manipulator and one slave robot manipulator. The master manipulator has two joints: one prismatic joint $q_{1}$ for moving the holding device up and down and one rotation joint $q_{2}$ for rotating the cell holding device. The slave manipulator has two prismatic joints $q_{3}$ and $q_{4}$ for moving well plates in the horizontal plane.

According to the Denavit-Hartenberg formulation [18], a coordinate system $\left(X_{i}, Y_{i}, Z_{i}\right)$ for $i=1,2,3,4, e$, is assigned to the $i_{\text {th }}$ link of the cooperative robot manipulator from base link to the end-effector, as shown in Fig. 4. The lower script $o$ represents the base link, and the lower script $e$ refers to the end-effector. The upper script $m$ refers to the master manipulator, and the upper script $s$ represents the slave manipulator. $l_{i}(i=1,2,3,4), d, n$, and $c$ are structural parameters of the cooperative mechanism. $l_{1}, l_{2}$, and $l_{3}$ are the structural parameters of the end-effector of the master manipulator. $l_{1}$ is the distance between $O_{1}$ and $O_{2} \cdot l_{2}$ and $l_{3}$ are used to define the origin location $O_{e}^{m}$ of the end-effector coordinate system $\left(X_{e}^{m}, Y_{e}^{m}, Z_{e}^{m}\right)$ of the master manipulator. The origin coordinate $O_{e}^{m}$ is expressed as $\left(0,-l_{2}, l_{3}\right)$ with reference to the coordinate system of the link 2. $l_{4}$ is the structural parameter of the end-effector of the slave manipulator, and is distance between the origin location $O_{e}^{s}$ of the end-effector coordinate system $\left(X_{e}^{s}, Y_{e}^{s}, Z_{e}^{s}\right)$ of the slave manipulator and the origin location $\mathrm{O}_{3}$ of the link 3 coordinate system $\left(X_{3}, Y_{3}, Z_{3}\right) . a, b$, and $c$ are used to define the origin coordinate $O_{0}^{s}$ of the slave base coordinate system $\left(X_{0}^{s}, Y_{0}^{s}, Z_{0}^{s}\right)$ as $(a, b,-c)$ with reference to the coordinate system $\left(X_{0}^{m}, Y_{0}^{m}, Z_{0}^{m}\right)$ of the master manipulator.

Using the homogenous transformation formulation, the kinematics of the cooperative robotic mechanism is:

$$
\begin{array}{r}
\left(\begin{array}{cc}
R_{s} & p_{s}\left(q_{3}, q_{4}\right) \\
0 & 1
\end{array}\right)=\left(\begin{array}{cc}
R_{m}\left(q_{2}\right) & p_{m}\left(q_{1}, q_{2}\right) \\
0 & 1
\end{array}\right) \\
\cdot\left(\begin{array}{cc}
R_{s}^{m} & p \\
0 & 1
\end{array}\right)
\end{array}
$$

where

$$
\begin{aligned}
R_{m}=\left(\begin{array}{ccc}
\cos q_{2} & 0 & \sin q_{2} \\
0 & 1 & 0 \\
-\sin q_{2} & 0 & \cos q_{2}
\end{array}\right), \quad R_{s}=\left(\begin{array}{lll}
1 & 0 & 0 \\
0 & 1 & 0 \\
0 & 0 & 1
\end{array}\right) \\
p_{m}=\left(\begin{array}{c}
-l_{2} \sin q_{2} \\
l_{1}+l_{3} \\
q_{1}-l_{2}+l_{2} \cos q_{2}
\end{array}\right), \quad p_{s}=\left(\begin{array}{c}
d-q_{4} \\
n-q_{3}-l_{4} \\
-c
\end{array}\right)
\end{aligned}
$$

$R_{s}^{m}$ represents the pose of the coordinate system $X_{e}^{s}, Y_{e}^{s}, Z_{e}^{s}$ and is determined by the cooperative manipulation task. Equation (1) was used to determine the structure parameters and control the joint motions for achieving the zebrafish embryo transfer task.

\section{System Setup}

The overall system is shown in Fig. 5(a). Fig. 5(b) shows a picture of the experimental setup. A water pump was used for loading culture media (or water) into the wells of the cell holding device. A precision vacuum pump system was in-house developed for trapping and releasing zebrafish embryos as well as for collecting redundant water and embryos. The master manipulator was constructed with a linear stage (New Mark, ET-100-10, travel range: $100 \mathrm{~mm}$, resolution: $0.04 \mu \mathrm{m}$ ) and a rotation stepper motor (Anaheim Automation, 15Y102S-LW4, resolution: $0.225^{\circ}$ ). A standard $\mathrm{X}-\mathrm{Y}$ motorized stage (Prior Scientific, H117, travel range: $114 \mathrm{~mm} \times 76 \mathrm{~mm}$, resolution: $0.01 \mu \mathrm{m}$ ) was used as the slave manipulator. The controllers of the two manipulators and a vision system (see next section) of two CMOS cameras (Basler, A601f) communicated with a host computer.

\section{VISION-GUIDED ALIGNMENT}

To deposit embryos into wells of a multiwell microplate, the position and the orientation between the holding device and the microplate must be calibrated and aligned. Error tolerances are summarized in Table I. A coordinate system is defined for calibration and alignment, as shown in Fig. 6(a). Fixture 1 is used to fix the microplate to the slave manipulator, and Fixture 2 is used to attach the cell holding device to the master manipulator. A block was manufactured and attached on Fixture 1. Alignment is conducted based on Fixtures 1 and 2. In Fig. 6(a), $A_{1} O_{1} B_{1}$ is the outside corner of the block, and $A_{2} O_{2} B_{2}$ is the outside 
(a)

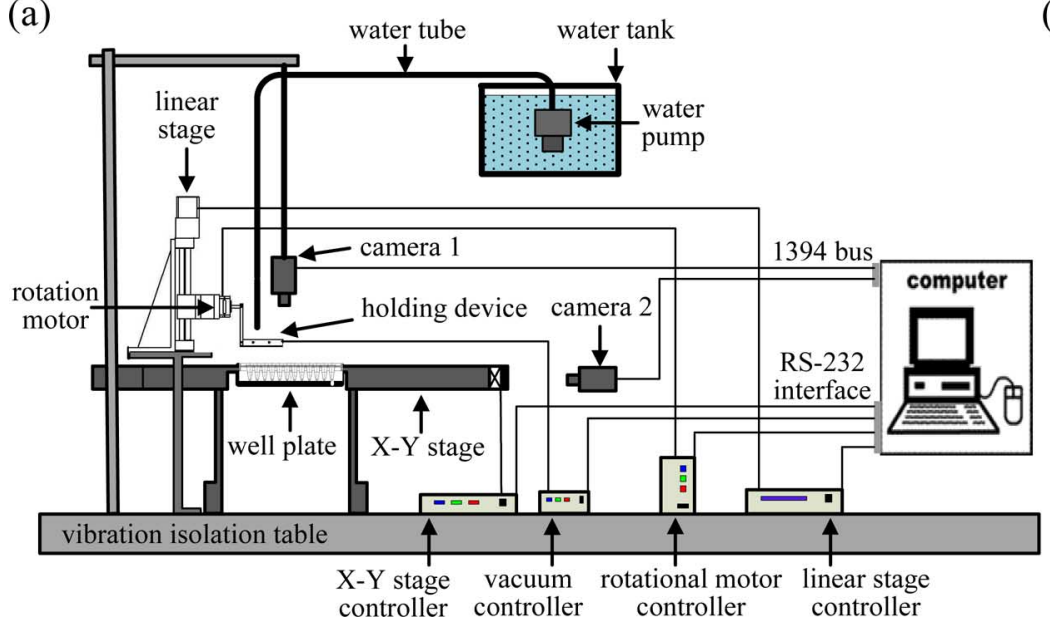

(b)

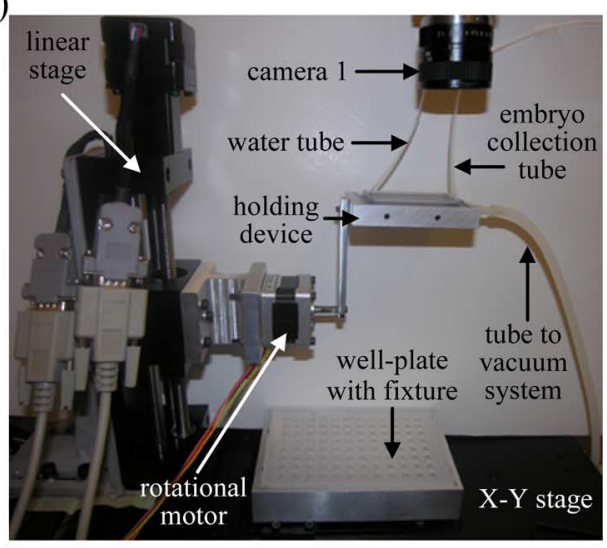

Fig. 5. System setup. (a) Schematic diagram of experimental system setup. (b) Photo of part of the system setup.

corner of Fixture 2. The top surface (determined by $A_{1} O_{1} B_{1}$ ) of the block and the top surface (determined by $\mathrm{A}_{2} \mathrm{O}_{2} \mathrm{~B}_{2}$ ) of Fixture 2 are located in the horizontal plane (e.g., $X O Y$ plane), $O_{1} A_{1}$ is designed to be parallel to $O_{2} A_{2}$ with distance $a_{0}$, and is designed to be parallel $O_{1} B_{1}$ to $O_{2} B_{2}$ with distance $b_{0}$ when the cell holding device and region I on the microplate are aligned, as shown in Fig. 6(a).

The orientation between the cell holding device and the microplate around $Z$ or $X$ axis was manually calibrated using a spirit level (RABO, aluminum spirit level), which has an angle accuracy of $0.057^{\circ}$. This calibration is conducted when the robotic mechanisms is installed or assembled. The orientation around $Y$ axis and the position along $X, Y$, and $Z$ axis are aligned during system operation using vision guidance.

The slave manipulator ( $X-Y$ stage) moves in the horizontal plane $\left(A_{1} O_{1} B_{1}\right)$, which is taken as a reference for alignment. Camera 1 is installed perpendicular to the motion plane of the slave manipulator [Fig. 5(a)]. The camera's position is adjusted such that the focus of the camera is located in the $A_{1} O_{1} B_{1}$ plane. Camera 2, as shown in Fig. 5(a), is installed in the horizontal plane along the negative $Y$ axis to focus on $A_{1} O_{1}$. The installation orientation of the cameras is calibrated with a spirit level with an accuracy of $0.057^{\circ}$.

To conduct the alignment of the orientation around $Y$ axis and the position along $Z$ axis, an image of the outside corners of Fixtures 1 and 2 is taken by camera 2. The edges of corners ( $A_{1} O_{1}$ and $A_{2} O_{2}$ ) are extracted by Hough line transform. The angle $\beta$ [Fig. 6(b)] and height $h$ between the two edges are calculated. The joint motion of the master manipulator is adjusted accordingly to reduce $\beta$ and $h$ to zero so that the orientations of the holding device and the microplate around the $Y$ axis are aligned and located at the same level. Similarly, to conduct the alignment of the position along $X$ and $Y$ axis, the top image of the outside corners of Fixtures 1 and 2 is taken by camera 1 . The edges of each corner $\left(A_{1} O_{1} B_{1}\right.$ and $\left.A_{2} O_{2} B_{2}\right)$ are detected. The distance $a$ and $b$ in the horizontal plane between the two fixtures is calculated and fed back to the slave manipulator to make $a$ equal to $a_{0}$, and $b$ equal to $b_{0}$ [Fig. 6(c)]. The orientation and position accuracies, summarized in Table I, satisfied the requirements for batch embryo transfer. It takes less than $5 \mathrm{~s}$

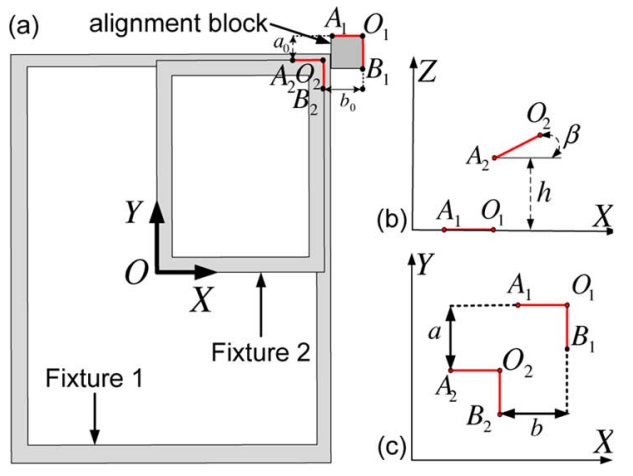

Fig. 6. Schematic of alignment strategy. (a) Alignment of the cell holding device and the microplate. (b) Alignment with camera 2. (c) Alignment with camera 1 .

TABLE I

CALibration AND Alignment Results

\begin{tabular}{cccc}
\hline $\begin{array}{c}\text { Orientation/Position } \\
\text { Alignment }\end{array}$ & $\begin{array}{c}\text { Error } \\
\text { Tolerance }\end{array}$ & $\begin{array}{c}\text { System } \\
\text { Accuracy }\end{array}$ & $\begin{array}{c}\text { Alignment } \\
\text { Method }\end{array}$ \\
\hline Orientation around Z & $1^{\circ}$ & $0.057^{\circ}$ & Spirit Level \\
Orientation around X & $1.9^{\circ}$ & $0.057^{\circ}$ & Spirit Level \\
Orientation around Y & $3.0^{\circ}$ & $1.8^{\circ}$ & Vision \\
Position along Z & $0.5 \mathrm{~mm}$ & $20 \mu \mathrm{m}$ & Vision \\
Position along Y & $1.3 \mathrm{~mm}$ & $20 \mu \mathrm{m}$ & Vision \\
Position along X & $1.3 \mathrm{~mm}$ & $20 \mu \mathrm{m}$ & Vision \\
\hline
\end{tabular}

to finish alignment of the orientation around $Y$ axis and the position along $Z$ axis, and $2 \mathrm{~s}$ to finish position alignment along $X$ and $Y$ axis.

\section{EMBRYO TRANSFER}

\section{A. Embryo Trapping}

Adhesion forces, particularly the capillary force [19]-[22] dominate the gravitational force at small scales, which is true in the case of zebrafish embryo manipulation. To match dimensions of standard 96-well microplates, through-hole pitch of the cell holding device is set to be $9 \mathrm{~mm}$. Due to the sparse spacing of through-holes, adhesion forces among embryos and adhesion forces between embryos and the substrate make it difficult for 


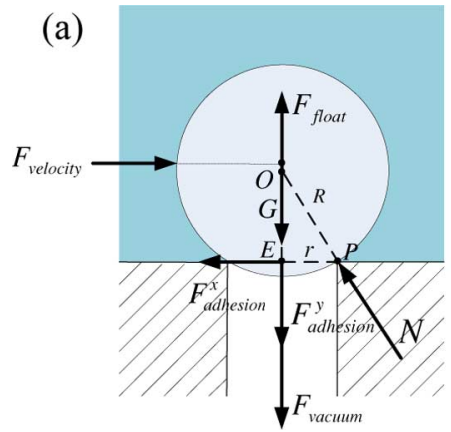

(b)

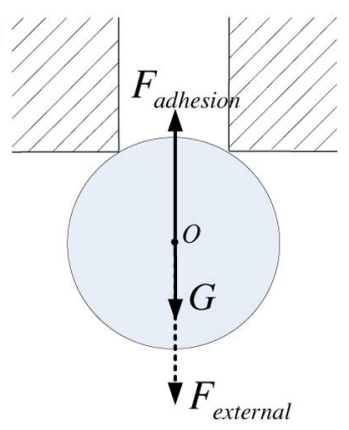

Fig. 7. Force analysis. (a) During embryo trapping, embryo faces up. (b) During embryo release, embryo faces down.

embryos to disperse themselves close to the through-holes on the cell holding device, even when vacuum is applied. Embryos often form clumps and stay in corners of the cell holding device.

To tackle the adhesion issue, water is continuously applied with the water pump, and the cell holding device is vibrated by the master manipulator. Water/culture medium provided by the water pump keeps embryos submerged in a liquid environment, and hence, significantly reduces adhesion among embryos and between embryos and the substrate. Water is pumped at a low flow rate, helping move embryos slightly; however, it is not strong enough to fully spread embryos close to the through-holes. Through vibrating (rotating) the cell holding device at a low frequency of $2-5 \mathrm{~Hz}$ using the master manipulator, liquid flow is generated, and a velocity pressure (force) is applied on the embryos. The force due to velocity moves embryos back and forth and moves them close to the through-holes. The rotation velocity and vacuum pressure are chosen according to force analysis of an embryo at equilibrium [Fig. 7(a)]

$$
\begin{aligned}
F_{\text {vacuum }}=\frac{2 R-\sqrt{R^{2}-r^{2}}}{r} & F_{\text {velocity }} \\
& +F_{\text {float }}-F_{\text {adhesion }}-G
\end{aligned}
$$

where $F_{\text {vacuum }}$ is proportional to applied vacuum pressure, $F_{\text {velocity }}$ is the force due to velocity, which increases with the rotation velocity of the cell holding device (note that force due to velocity caused by the pumped water is low and negligible), $F_{\text {float }}$ is the buoyancy force, $F_{\text {adhesion }}$ is the adhesion force between the embryo and substrate, $G$ is gravity of the embryo, $R$ is the radius of the embryo, and $r$ is the radius of the through-hole. Note that $F_{\text {velocity }}$ is the force caused by dynamic pressure $P_{\text {velocity }}$ [23]. The dynamic pressure is generated from the movement of liquid and can be expressed as $P_{\text {velocity }}=(1 / 2) \rho v^{2}$, where $\rho$ is fluid density, and $v$ fluid velocity. To simplify force analysis, derivation of (2) was based on the rigid model of embryos. In experiments, the applied pressure needs to be adjusted with the consideration of the effect of embryo deformation generated by the vacuum pressure. It can be seen from (2), a larger rotation velocity of the cell holding device requires a larger vacuum pressure applied to maintain equilibrium. Since high vacuum pressures undesirably over-deform embryos, both vacuum pressure and rotational speed of the cell holding device were chosen to be

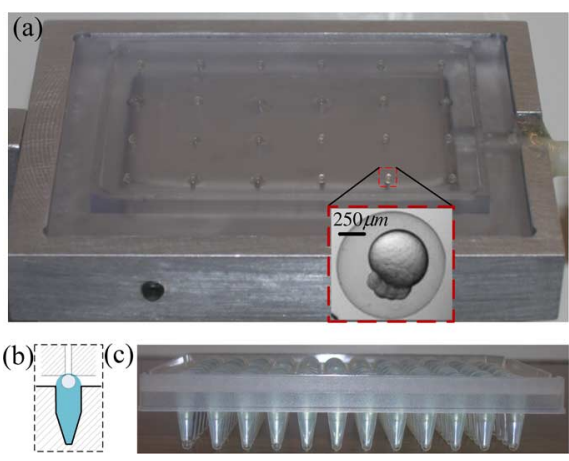

Fig. 8. (a) Twenty-four zebrafish embryos were trapped on the cell holding device. (b) Schematic diagram of releasing a embryo (white circle) into a liquid environment (semi-sphere droplet). (c) Picture of semi-sphere droplets on top of wells of a microplate.

low but sufficient to overcome the adhesion force for efficiently spreading/dispersing the embryos to the through-holes of the device. In experiments, the rotation angle velocity of the cell holding device was chosen to be $20-30 \%$ s with a magnitude of $3^{\circ}$. The vacuum pressure was chosen to be $1.5-3.5 \mathrm{kPa}$. It takes less than 30 s to successfully trap 6 by 4 embryos on the cell holding device [Fig. 8(a)].

\section{B. Embryo Release}

Force analysis of an embryo at equilibrium [Fig. 7(b)] is expressed

$$
F_{\text {external }}=F_{\text {adhesion }}-G \text {. }
$$

To transfer zebrafish embryos from the cell holding device into the wells of a microplate, zebrafish embryos need to be released from the cell holding device [Fig. 7(b)]. Adhesion forces between embryos and the cell holding device make release difficult since embryos tend to tightly adhere to the device even when the device is rotated upside down to face wells on the microplate. To achieve embryo release, one can either reduce the capillary force until it is less than the gravity of a embryo or apply external forces to overcome the capillary force.

The first approach experimented in this work is to introduce a liquid environment to eliminate or decrease the capillary force. The capillary force is generated at the surface between liquid and air. The strategy was implemented by generating a semisphere water droplet on the top of each well of the microplate, as shown in Fig. 8(c). In this work, semi-sphere droplets were formed by filling each well with $400 \mu l$ culture medium using a pipette manually, which can be readily conducted by a standard liquid handling robot. The height of semi-sphere droplet is around $3 \mathrm{~mm}$. With half-sphered culture medium, embryos are released in a liquid environment when the cell holding device is moved downwards close to the well-plate by the master manipulator.

The second approach is to apply positive pressure to repel embryos from the cell holding device for release [i.e., applying an external force $F_{\text {external }}$ to overcome the adhesion force, shown in Fig. 7(b)]. Challenge is to properly control pressure for release. The difficulty of proper pressure application will be discussed in more detail in Section V. 
The third strategy is to accelerate (or vibrate) the embryos by controlling the motion of the device using the system. Accelerating the holding device generates an external force (inertial force) on each embryo. When the inertial force plus gravity of an embryo is larger than the adhesion force, the embryo can be released into the well under it. According to (3), the minimum acceleration of the device is $\left(F_{\text {adhesion }}-G\right) / m$ to release trapped embryos. According to (3), the minimum acceleration of the device is $\left(F_{\text {adhesion }}-G\right) / m$ to release trapped embryos. In this work, a linear stage is used to vibrate the cell holding device. An acceleration of $20 \mathrm{~m} / \mathrm{s}^{2}$ was applied to the device with a magnitude of $3 \mathrm{~mm}$. Traditional stepper motors are not able to generate high accelerations and must be combined with other solutions (e.g., a liquid environment) to release trapped cells. The use of a piezoelectric or supersonic motor, which produces high accelerations, can possibly be more efficient for embryo release.

\section{Operation Flow of Embryo Transfer}

Before embryo transfer, the system performs an alignment procedure, as described in Section III. To start the operation, the cell holding device is rotated to face upwards by the master manipulator. Each well of the microplate is filled with culture medium to form a semi-sphere water droplet on the top of each well. In this proof-of-principle study, embryos are loaded onto the cell holding device using a micropipette, which can be automated with a water or air pump system. The cell holding device is vibrated by rotating it back and forth with small magnitude to fully spread the embryos on the device. In the meanwhile, a negative pressure is applied to trap the embryos on top of the through-holes. Redundant embryos are manually collected from the device while culture medium is continuously provided. These excess embryos are used in the next operation of batch transfer. The excess embryos are collected manually using a pipette in this work. Automation can be fulfilled by integrating an air pump system for excess embryo collection. Alternatively, it is also possible to dump excess embryos and medium into a movable collection tank when the holding device is flipped upside down. The cell holding device is rotated to face downward and is lowered until the embryos are fully submerged inside the semi-sphere water droplet. The negative pressure is then turned off. The cell holding device is accelerated (vibrated) by moving it up and down with small magnitude to assist the release of embryos into wells of the microplate. The microplate is positioned by the slave manipulator to position region II under the cell holding device. The above procedure is repeated until all wells of the microplate are filled with embryos.

\section{EXPERIMENT RESULTS AND DISCUSSIONS}

Adhesion forces between embryos and the cell holding device make it difficult to release embryos into target wells. As summarized in Table II, experiments confirmed that providing a liquid environment (Case A) is necessary for embryo release. Without providing a liquid environment, applying positive pressure only (Case B) cannot release embryos effectively due to the difficulty of applying proper pressures. Adhesion forces on embryos within the same batch are different due to varied sizes of the embryos and their physical properties. Therefore, in Case $\mathrm{B}$, embryos are not released at the same time instantly, leading
TABLE II

EXPERIMENTAL RESULTS CLASSIFIED INTO FIVE CASES

\begin{tabular}{cccc}
\hline $\begin{array}{c}\text { Experiment } \\
\text { Strategy }\end{array}$ & $\begin{array}{c}\text { Number of Embryos } \\
\text { Transferred }\end{array}$ & $\begin{array}{c}\text { Number of } \\
\text { Wells with One } \\
\text { Embryo }\end{array}$ & $\begin{array}{c}\text { Success } \\
\text { Rate }\end{array}$ \\
\hline A & 120 & 72 & $60 \%$ \\
B & 24 & 4 & $16.9 \%$ \\
C & 24 & 0 & 0 \\
D $(\mathrm{A}+\mathrm{B})$ & 48 & 22 & $45.8 \%$ \\
E $(\mathrm{A}+\mathrm{C})$ & 1056 & 996 & $94.3 \%$ \\
\hline
\end{tabular}

TABLE III

Detailed Results of CASE E

\begin{tabular}{|c|c|c|c|c|c|c|}
\hline \multirow[t]{2}{*}{$\begin{array}{l}\text { Well-plate } \\
\text { Number }\end{array}$} & \multicolumn{4}{|c|}{$\begin{array}{c}\text { Number of Wells with } \\
\text { One Embryo for Each } \\
\text { Region }\end{array}$} & \multirow[t]{2}{*}{$\begin{array}{c}\text { Number of } \\
\text { Wells with } \\
\text { Two Embryos }\end{array}$} & \multirow[t]{2}{*}{$\begin{array}{c}\text { Success } \\
\text { Rate }\end{array}$} \\
\hline & I & II & III & IV & & \\
\hline 1 & 23 & 24 & 23 & 22 & 0 & $95.8 \%$ \\
\hline 2 & 24 & 23 & 24 & 23 & 0 & $97.9 \%$ \\
\hline 3 & 23 & 22 & 22 & 22 & 2 & $92.7 \%$ \\
\hline 4 & 22 & 23 & 23 & 23 & 1 & $94.8 \%$ \\
\hline 5 & 21 & 22 & 22 & 23 & 2 & $91.7 \%$ \\
\hline 6 & 21 & 22 & 22 & 24 & 1 & $92.7 \%$ \\
\hline 7 & 21 & 24 & 22 & 23 & 1 & $93.8 \%$ \\
\hline 8 & 23 & 23 & 24 & 23 & 0 & $96.9 \%$ \\
\hline 9 & 22 & 23 & 22 & 21 & 2 & $91.7 \%$ \\
\hline 10 & 22 & 22 & 23 & 21 & 1 & $91.7 \%$ \\
\hline 11 & 22 & 24 & 24 & 24 & 0 & $97.9 \%$ \\
\hline
\end{tabular}

to pressure interactions between neighboring through-holes. An embryo can escape through the clearance between the device and the microplate, or change its motion direction rather than traveling vertically as desired. Consequently, some wells were filled with more than two embryos, and most wells were empty.

When only acceleration is generated (Strategy C in Table II) without providing a liquid environment, success rate of embryo release was zero. In our system setup, the traditional stepper motor is not able to generate sufficient acceleration. The use of a piezoelectric or supersonic motor might produce sufficient acceleration for embryo release. However, the acceleration method in this work can only be used to assist release and must be combined with other solutions for embryo release in order to produce a high success rate. Case D in Table II refers to a combined use of strategy A with strategy B. Approximately $25 \%$ of the wells had more than one embryo after embryo release, demonstrating that positive pressure application is not suitable for single embryo transfer.

When a liquid environment is provided, and embryo release is assisted with acceleration of the cell holding device (Case E in Table II), out of the 1056 zebrafish embryos used in experiments (i.e., 44 times parallel transfer into 11 96-well plates), 996 zebrafish embryos successfully reached desired wells, amounting to a success rate of $94.3 \%$. These experiments demonstrate that a combined use of liquid environment and acceleration is the most effective for zebrafish embryo transfer. Detailed results for each of the 11 microplates are summarized in Table III. Zebrafish embryos transferred into one of microplates were cultured at $28.5{ }^{\circ} \mathrm{C}$ and all developed into zebrafish (Fig. 1). The $100 \%$ development rate confirms that the embryo trapping and release process is safe on zebrafish embryos without causing significant damage.

As listed in Table III, in those wells where embryo transfer failed, 51 out of the 1056 wells were empty; and 9 of the 
1056 wells had two embryos. The first reason for the failures is possibly due to embryo quality variations. For example, a low number of embryos had abnormal morphology and possibly lower stiffness that can cause too large deformations into the through-holes on the cell holding device during embryo trapping. This physical property abnormality can also cause difficulty in embryo release. Better embryo quality control would reduce this failure.

The second possible reason is that semi-sphere droplets on top of the microplate were formed manually with a pipette due to the lack of a liquid handling robot in our lab. The imperfection and inconsistency (e.g., semi-sphere droplets were different in height) made some of the droplets contacted earlier than others by embryos and the cell holding device. These droplets flowed to neighboring wells and can carry embryos into the neighboring wells. On the other hand, some of the semi-sphere droplets were too low and did not make the embryos submerged into the droplets, causing failure of embryo release. When a liquid handling robot is used for droplet formation, this problem would be mitigated.

The third reason is that bubbles could have occurred in some wells when the wells were filled with culture medium. Disappearance of bubbles during embryo transfer can cause the liquid level to decrease and thus, affect embryo release. This severity of the problem can be reduced with the use of a standard liquid handling robot. Finally, machining accuracy of the in-house made cell holding device and mechanism constructed for this feasibility study was limited. Machining errors can cause the height of the clearance between the cell holding device and the 96-well microplates to be nonequal.

The system takes averagely $90 \mathrm{~s}$ to fill 24 wells of a 96-well microplate with one zebrafish embryo per well: $30 \mathrm{~s}$ for embryo trapping and $60 \mathrm{~s}$ for release. The long release time ensures that all embryos are released from the vibrations produced by the master manipulator, which can be reduced with more uniform semi-spherical droplets dispensed. Compared to the only commercial system (COPAS XL, Unioin Biometica), which costs $120 \mathrm{~s}$ to fill a 96-well plate with one embryo per well, the speed produced by our prototype system is three times slower. The operation time spent on embryo release can be significantly decreased when an additional piezoelectric or supersonic motor is employed to generate a high acceleration during the vibration of the cell holding device. The high acceleration of cells can efficiently overcome the adhesion between cells and the substrate. Therefore, the operation speed can be further improved when the cell holding device is scaled up (e.g., containing 96 throughholes) and system components are more accurately constructed.

\section{CONCLUSION}

This paper presented a prototype corporative robotic system for transferring zebrafish embryos into standard 96-well microplates. The cell holding device has through-holes matching the configuration of microplates. Trapped embryos are robotically released into multiple wells in parallel. The robotic system has four degrees-of-freedom, responsible for moving and aligning the cell holding device and a microplate. The system transferred 1056 zebrafish embryos into 11 96-well plates, demonstrating a success rate of $94.3 \%$. This preliminary study proved the principle of parallel transfer of zebrafish embryos using a robotic approach.

\section{REFERENCES}

[1] C. Parng, W. L. Seng, C. Semino, and P. Mcgrath, "Zebrafish: A preclinical model for drug screening," ASSAY and Drug Development Technol., vol. 1, no. 1, pp. 41-48, 2002.

[2] C. K. Kaufman, R. W. White, and L. Zon, "Chemical genetic screening in the zebrafish embryo," Nature Protocols, vol. 4, no. 10, pp. 1422-1432, 2009.

[3] N. Mandrekar and N. L. Thakur, "Significance of the zebrafish model in the discovery of bioactive molecules from nature," Nature Biotechnol. Lett., vol. 31, no. 2, pp. 171-179, 2009.

[4] A. Smith, "Screening for drug discovery: The leading question," $\mathrm{Na}$ ture, vol. 418, pp. 453-459, 2002.

[5] T. Chapman, "Lab automation and robotics: Automation on the move," Nature, vol. 421, pp. 661-666, 2003.

[6] J. G. Houston, "The impact of robotics and novel assay technologies on lead discovery processes," Pharmacochem. Library, vol. 29, pp. 191-202, 1998 .

[7] A. Sparkes, R. D. King, W. Aubrey, M. Benway, E. Byrne, A. Clare, M. Liakata, M. Markham, K. E. Whelan, M. Young, and J. Rowland, "An integrated laboratory robotic system for autonomous discovery of gene function," J. Assoc. Lab. Autom., vol. 15, no. 1, pp. 33-40, 2010.

[8] A. Vogt, A. Cholewinski, X. Shen, S. G. Nelson, J. S. Lazo, M. Tsang, and N. A. Hukriede, "Automated image-based phenotypic analysis in zebrafish embryos," Developmental Dynamics, vol. 238, no. 3, pp. 656-663, 2009.

[9] K. A. Dehring, H. L. Workman, K. D. Miller, A. Mandagere, and S. K. Poole, "Automated robotic liquid handling/laser-based nephelometry system for high throughput measurement of kinetic aqueous solubility," J. Pharmaceutical Biomed. Anal., vol. 36, no. 3, pp. 447-456, 2004.

[10] M. J. Felton, "Liquid handling: Dispensing reliability," Anal. Chem., vol. 75, no. 17, pp. 397-399, 2003.

[11] R. D. Murphey and L. I. Zon, "Small molecule screening in the zebrafish," Methods, vol. 39, no. 3, pp. 255-261, 2006

[12] J. P. Desai, A. Pillarisetti, and A. D. Brooks, "Engineering approaches to biomanipulation," Annu. Rev. Biomed. Eng., vol. 9, pp. 35-53, 2007.

[13] P. Jordan, J. Leach, M. Padgett, P. Blackburn, N. Isaacs, M. Goksr, D. Hanstorp, A. Wright, J. Girkin, and J. Cooper, "Creating permanent 3D arrangements of isolated cells using holographic optical tweezers," Lab on a Chip, vol. 5, no. 11, pp. 1224-1228, 2005.

[14] K. Ino, M. Okochi, N. Konishi, M. Nakatochi, R. Imai, M. Shikida, A Ito, and H. Honda, "Cell culture arrays using magnetic force-based cell patterning for dynamic single cell analysis," Lab on a Chip, vol. 8, no. 1, pp. 134-142, 2008.

[15] A. Mizuno, M. Imamura, and K. Hosoi, "Manipulation of single fine particle in liquid by electrical force in combination with optical pressure," IEEE Trans. Ind. Appl., vol. 27, no. 1, pp. 140-146, Jan./Feb. 1991.

[16] W. H. Wang, X. Y. Liu, and Y. Sun, "High-throughput automated injection of individual biological cells," IEEE Trans. Autom. Sci. Eng., vol. 6, no. 2, pp. 209-219, Apr. 2009.

[17] X. Y. Liu and Y. Sun, "Microfabricated glass devices for rapid single cell immobilization in mouse zygote microinjection," Bomed. Microdevices, vol. 11, no. 6, pp. 1169-1174, 2009.

[18] J. K. Davidson and K. H. Hunt, Robots and Screw Theory: Applications of Kinematics and Statics and Robotics. London, U.K.: Oxford Univ. Press, 2004.

[19] M. Savia and H. N. Koivo, "Contact micromanipulation-Survey of strategies," IEEE/ASME Trans. Mechatronics, vol. 14, no. 4, pp. 504-514, Aug. 2009.

[20] R. S. Fearing, "Survey of sticking effects for micro parts handling," in IEEE/RSJ Conf. Intell. Robot. Syst., Pittsburgh, PA, 1995, vol. 2, pp. 212-217.

[21] A. Torii, M. Sasaki, K. Hane, and S. Okuma, "Adhesive force distribution on microstructures investigated by an atomic force microscope," Sens. Actuators A, vol. A44, no. 2, pp. 153-158, 1994.

[22] M. Gauthier, S. Rgnier, P. Rougeot, and N. Chaillet, "Forces analysis for micromanipulations in dry and liquid media," J. Micromechatronics, vol. 3, no. 3-4, pp. 389-413, 2006.

[23] L. J. Clancy, Aerodynamics. London, U.K.: Pitman Publishing Limited, 1975. 


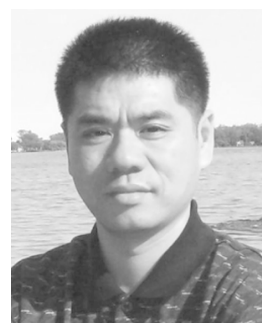

Xuping Zhang (M'11) received the B.Eng. degree in mechanical engineering from Chongqing University, Chongqing, China, in 1992, and the Ph.D. degree in mechanical engineering from the University of Toronto, Toronto, ON, Canada, in 2009.

$\mathrm{He}$ is currently a Postdoctoral Fellow in the Advanced Micro and Nanosystems Laboratory, Mechanical and Industrial Engineering Department, University of Toronto. His research interests include robotics, dynamics and control, vibration theory, and mechanisms.

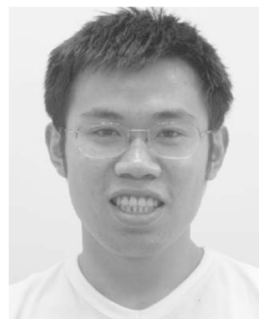

Zhe Lu (S'07-M'10) received the B.Eng. degree in control engineering from Harbin Institute of Technology, Harbin, China, in 2002, and the Ph.D. degree in mechanical engineering from the National University of Singapore, Singapore, in 2007.

$\mathrm{He}$ is currently a Postdoctoral Fellow in the Advanced Micro and Nanosystems Laboratory, Department of Mechanical and Industrial Engineering, University of Toronto, Toronto, ON, Canada. His research interests include robotics, automation, and mechatronics.

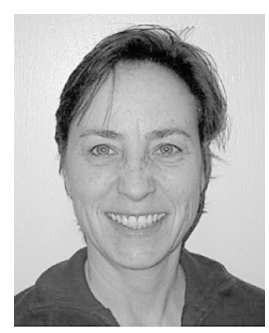

Danielle Gelinas received the Ph.D. degree in biology from Boston University, Boston, MA, in 1995, where her research focused on the neuroendocrine regulation of brain steroid metabolism in teleost fish.

She is currently a Laboratory Research Project Coordinator in the Developmental and Stem Cell Program at the Research Institute in the Hospital For Sick Children, Toronto, ON, Canada.

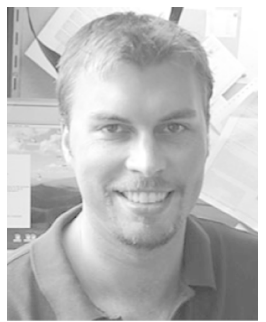

Brian Ciruna is a Scientist in the Developmental and Stem Cell Biology Program, Hospital for Sick Children, Toronto, ON, Canada. He is also an Assistant Professor in the Department of Molecular Genetics, and a faculty member in the Collaborative Program in Developmental Biology, University of Toronto.

Dr. Ciruna currently holds a Canada Research Chair in Developmental Genetics and Cell Biology.

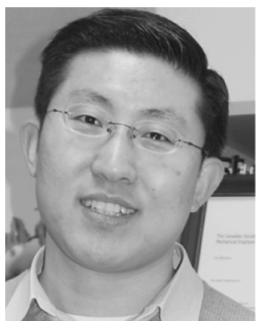

Yu Sun (S'01-M'03-SM'07) received the Ph.D. degree in mechanical engineering from the University of Minnesota, Minneapolis, in 2003.

$\mathrm{He}$ is an Associate Professor at the University of Toronto, Toronto, ON, Canada. Before joining the faculty of the University of Toronto in July 2004, he was a Research Scientist at the Swiss Federal Institute of Technology (ETH-Zrich). His research includes design and fabrication of MEMS/NEMS devices; micronanorobotic manipulation under optical and electron microscopes, and manipulation and characterization of biological cells, biomolecules, and nanomaterials. 